\title{
Life in the pressure cooker: The thermal unfolding of proteins from hyperthermophiles
}

\author{
H.H.Klump*, M.W.W.Adams**, and F.T.Robb+ \\ * Dept. of Biochemistry, University of Cape Town, \\ 7700 Rondebosch, RSA \\ ** Dept.Biochemistry, University of Georgia, Athens, Ga, USA \\ + Center of Marine Biotechnology, University of Maryland, \\ Baltimore, USA
}

\begin{abstract}
keported here are the first results of a quantitative thermodynamic study involving a series of proteins isolated from the anaerobic sulfur-reducing archaebacterium Pyrococcus furiosus which grows near subtiarine geothermal vents on the deep ocean floor. The proteins isolated and investigated here are the two non-heme iron proteins rubredoxin ( $\mathrm{Ww}$ 5400), and ferredoxin ( $\mathrm{Ww}$ 7500), and the enzyme glutamate dehydrogerase GDH (NW 270000). The enzyme is inactive at $40^{\circ} \mathrm{C}$ and fully activated $a t ~ 30{ }^{\circ} \mathrm{C}$. Independent of their particular primary structure and independent of the molecular weight $(\mathrm{Nw})$ all three proteins investigated unfold at exactly the same temperature $\left(113^{\circ} \mathrm{C}\right)$ The experimentally observed transition temperature $\mathrm{Tm}$ coincides with the extrapolated conversion temperature $T^{*}$ (Privalov, Privalov \& Gill, Murphy and Gill, Freire and Murphy, $1-4)$ of $112^{\circ} \mathrm{C}$. It is readily accepted that at this temperature the hydrophobic interactions will cease to contribute to the overall stability of the ordered secondary and/or tertiary structure causing the core of any globular protein to unfold to the denatured state. Thus $113^{\circ} \mathrm{C}$ seems to be the threshold temperature for sustantation of life on earth.
\end{abstract}

\section{INTRODUCTION}

The DNA taxonomy of the bacterial tree shows that the most ancestral bacteria are thermospheric and sulfur-loving. The so-called hyperthermophiles are a diverse group, although all of them are Archaea, formerly known as Archaebacteria (5-7). These bacteria form the base of a complex food chain that culminates in a multitude of exotic crabs, fishes and up to three feet long tube worms with no guts (8). A brief look at the ecology of hydrothermal-vent communities of the deep sea shows that besides the solar powered life at the upper ocean layers and on the earth's surface there is an other source for life sustaining energy which is entirely geothermally driven (9).

Studying life from this source is not only rewarding in terms of unraveling the history of all life on earth but it will also turn out to be most profitable in teaching us the rules for the design of proteins which exhibit drastically increased temperature stability. Another fascinating aspect of studying the hyperthermophilic bacteria and their proteins is to find out about the possibility of a temperature limit for protein stability in an aqueous environment.

Finally the experimental results will enable us to asses the merits of the various theoretical approaches to predict and explain protein stability. Privalov and Khechiashvili (10) were the first to notice from their rich experience in calorimetry of globular proteins that the enthalpy and entropy changes obtained for the denaturation of globular proteins converge at some characteristic temperature at or slightly above 
$100^{\circ} \mathrm{C}$ when they were normalized with respect to the number of residues per protein. Baldwin (11) could explain this convergence behavior convincingly at least as far as the entropy change is concerned by linking it to the hydrophobic effect. He observed that there is a striking similarity between the convergence temperature $\left(112^{\circ} \mathrm{C}\right)$ at which the entropy of unfolding of a globular protein becomes zero and the temperature at which the entropy of transfer of liquid hydro-carbons to water vanishes.

Initially it was assumed that both the convergence temperatures for the transition enthalpy $T * H$ and for the transition entropy $T * S$ are identical. Later it became apparent, especially from the results obtained by the group-additivity approach introduced by Murphy and Gil1 (3) that this is not quite true. A comparative study of the thermodynamic behavior of proteins and of the thermodynamics of dissolution of solid model compounds by these authors showed convincingly that the convergence temperature for the enthalpy and the entropy changes ( $T * H$ and $T * S$ respectively) corresponds to the temperature at which the apolar contribution to the enthalpy and entropy change become zero. It became apparent from their analysis that $\mathrm{T} * \mathrm{H}$ was about $100^{\circ} \mathrm{C}$ and $\mathrm{T} * \mathrm{~S}$ was more likely to be at about $112^{\circ} \mathrm{C}$. The polar contributions can be viewed as temperature independent and sequence independent, i.e. the contribution per residue is constant, while the apolar contribution varies.

Lee (12) choose a slightly different approach in the way that he expressed the thermodynamic parameters in terms of the area which will be exposed to the solvent water when the protein unfolds. His conclusion can be summed up to the statement that convergence is linked to the temperature at which polar and apolar contributions to the enthalpy of unfolding and the entropy change associated with this process become equal. If one takes into account that Murphy and Gill normalized the thermodynamic data with respect to the number of residues per protein while Lee's analysis is based on a normalization with respect to the total surface area exposed upon unfolding it becomes obvious that both approaches express the same finding. The experimental method of choice to test the validity of the predictions is microcalorimetry. We have investigated the thermal stability of three different proteins, namely rubredoxin (RdPf), ferredoxin (FdPf) and glutamin dihydrogenase (GDHPf) isolated from Pyrococcus furiosus. A fourth protein, the amylase (Ampf), was investigated by Privalov in cooperation with Ch. Anfinsen (private communication) (13). All four proteins investigated so far unfold at exactly the same temperature of $113^{\circ} \mathrm{C}$ irrespectively of all the differences in size and sequence composition.

\section{MATERIALS AND METHODS}

The GDH was purified from the cytoplasmic fraction of a cell free extract of $P$.furiosus. The protein was purified to homogeneity by chromatography on ion exchange, nuclear exclusion, and hydrophobic interaction media. $\mathrm{NADH}$ and NADPH were recognized with high affinity. The purified native protein was shown to be a hexameric complex (14) assembled from identical subunits with a molecular weights of $270 \mathrm{kilodalton.} \mathrm{The} \mathrm{half-life} \mathrm{of} \mathrm{the}$ enzyme is $3.5 \mathrm{~h}$ at $100^{\circ} \mathrm{C}$, which makes it the most thermostable enzyme reported to date. Both ferredoxin FdPf and rubredoxin RdPf from $P$.furiosus were isolated according to a standard protocol.

We have employed two independent physical methods, UV-melting recorded at $280 \mathrm{~nm}$ with the aid of a modified Pye Unicam P1800 spectrophotometer equipped with a pressurized thermostated cell compartment, and differential scanning microcalorimetry (DSC) (15) in a DASM-4 microcalorimeter. The sample compartment of this instrument can also be pressurized up to 4 bar. The heating rate during all scans was 1 deg. per minute. The pressure applied was large enough to shift the boiling temperature of the solvent water up to $160^{\circ} \mathrm{C}$ but low enough to avoid interference with protein stability. Glycylglycine buffer was chosen as to minimize pH shifts during the temperature rise (5). 

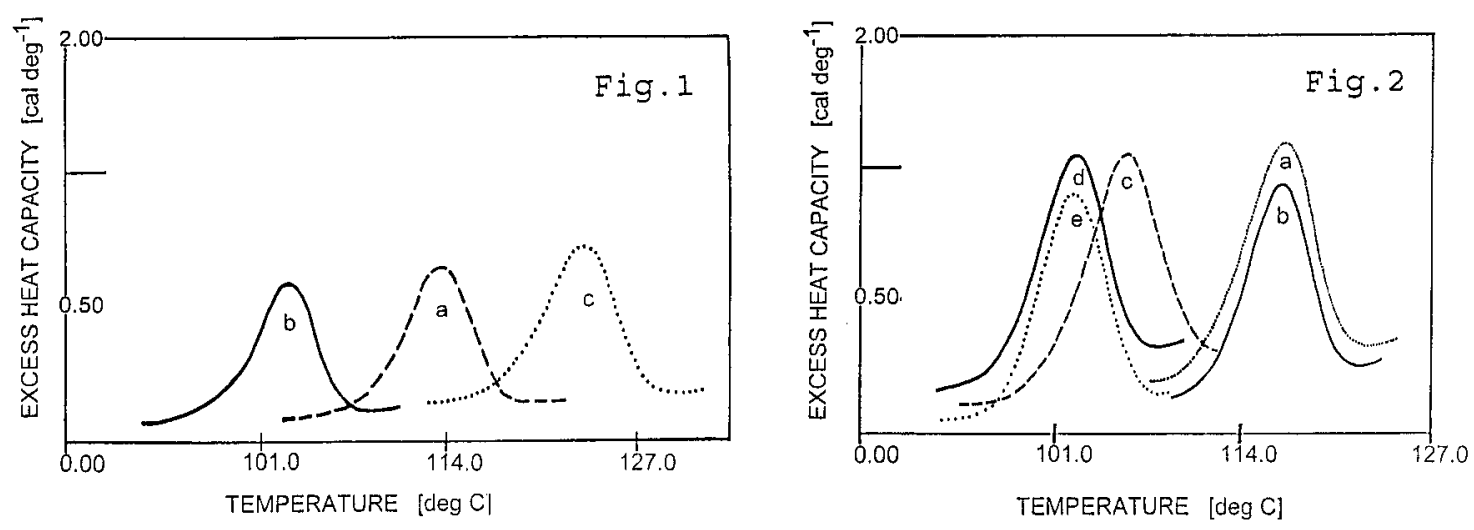

Fig.1 shows the excess heat capacity vs. temperature scans for the three different rubredoxin samples. The corresponding scans for the five ferredoxins are given in Fig. 2 .

\section{RESULTS AND DISCUSSION}

Giutamate-dehydrogenase (GDH):The results obtained from the two independent physical methods are complementary and can be summed up as follows: In the temperature interval between $45^{\circ} \mathrm{C}$ and $809_{\mathrm{C}}$ a small but significant cooperative increase in the absorbance signal ( $A_{280}$ ) was observed. This increase is complemented by a rather broad peak in the heat capacity vs. temperature curve. Both physical changes correspond to the same gradual loosening up of the room temperature structure, which has no enzymatic activity below $40^{\circ} \mathrm{C}$. Some unfolding has obviously to occur to allow for the onset of enzymatic activity. The threshold temperature for this activation is $45^{\circ} \mathrm{C}$. The full activity is only available at $80^{\circ} \mathrm{C}$, and it decreases again to zero on cooling the enzyme back to room temperature. This thermal activation step is a common feature of several proteins isolated from mesophilic bacteria. The advantage of this particular behavior is easy to understand if one considers the habitat of these bacteria and the steep temperature gradients in their marine environment where they drift in and out of hotter nutrition loaded vents. When the bacteria drift out of the vent they simply shut off their activities and turn them on again when the temperature becomes suitable. Drifting into a range of very hot water (up to $110^{\circ} \mathrm{C}$ ) does not harm the bacterium either. As can be shown from the calorimetric experiments heating the protein up to $112^{\circ} \mathrm{C}$ and rapidly cooling it back down to $80^{\circ} \mathrm{C}$ retains the native conformation. Only when the denaturation temperature of $113^{\circ} \mathrm{C}$ is surpassed the protein unfolds irreversibly, and the unfolded conformer tends to aggregate by the time taking the aggregate out of the denaturation/renaturation equilibrium. only in this respect is the thermal unfolding of GDH an irreversible step.

All the relevant thermodynamic data of the heat activation and the denaturation of GDHPf are listed in Table 1.

Table 1

\begin{tabular}{|llccccc|}
\hline & $\Delta \mathrm{H}_{\mathrm{Cal}}$ & $\Delta \mathrm{S}_{\mathrm{Cal}}$ & $\Delta \mathrm{H}_{\mathrm{VH}}$ & $\mathrm{T}_{\mathrm{L}}$ & $\mathrm{T}_{\mathrm{H}}$ & $\mathrm{n}$ \\
\hline Deanaturation & 414 & 1072 & 240 & & 113 & 1.7 \\
\hline Activation & 187 & 520 & 39.5 & 55 & & 4.8 \\
\hline
\end{tabular}


The ratio of $\Delta \mathrm{Hcal}$ over $\Delta \mathrm{H}$ van't Hoff equals 4.8 for denaturation and 1.7 for heat activation, indicating that neither activation nor denaturation is a simple two-state process. Values for $\Delta \mathrm{Hcal}$ and $\Delta$ Scal are the molar enthalpy and entropy changes, and are given in $\mathrm{Kcal} / \mathrm{mol}-1$, and $\mathrm{Kcal} / \mathrm{mol}-1$ $\mathrm{K}^{-1}$ respectively, assuming a molecular weight of 270.000 for the hexameric complex. IKcal equals $4.18 \mathrm{KJ}$.

It is obvious that heat activation is due to a marginal unfolding of the overall structure which is much less demanding in terms of energy input as compared to the total unfolding of a protein. Addition of the cofactor NADP does not increase the thermal stability of the GDH (5).

The other two proteins from the hyperthermophile Pyrococcus furiosus investigated by the same two methods are rubredoxin (RdPf), a protein which serves in the electron transfer chain of the bacterium, and ferredoxin FdPf) in the oxidized and the reduced form from Pf and from Thermotoga. The results of the DSC measurements are given in Table 2 .

Table 2

\begin{tabular}{lllll}
\hline \multicolumn{1}{c}{ Denaturation } & dHcal & $\Delta$ Scal & Tm & Fig. \\
\hline Rubredoxin (ox) & 22.5 & 57.0 & 113 & $1 \mathrm{a}$ \\
Rubredoxin (red.) & 20.2 & 53.8 & 102 & $1 \mathrm{~b}$ \\
Rubredoxin (Zn) & 23.8 & 60.0 & 123 & $1 \mathrm{c}$ \\
Ferredoxin (ox/4Fe) & 11.5 & 29.3 & 117.5 & $2 \mathrm{a}$ \\
Ferredoxin (red/4Fe) & 11.4 & 29.0 & 117.0 & $2 \mathrm{~b}$ \\
Ferredoxin (ox/3Fe) & 11.0 & 29.3 & 105 & $2 \mathrm{C}$ \\
Ferredoxin (therm*/ox/3Fe) & 11.0 & 29.3 & 102.5 & $2 \mathrm{~d}$ \\
Ferredoxin(therm/red/3Fe) & 11.0 & 29.3 & 102.0 & $2 \mathrm{e}$ \\
*Thermotoga & & & & \\
\hline
\end{tabular}

The structures of the oxidized and the reduced forms of rubredoxin from $P$. furiosus have been determined by $x$-rày crystallography to a resolution of $1.8 \AA$ by Day et al. (16). As outlined in their publication the improved stability can be understood as originating from three characteristic contribution, namely from improved main chain to main chain H-bonds, from changes in buried surface areas, and from amino acid residues unique to RdPf. Hyperthermostability is achieved with minimal alterations to the basic folding of RdPf. Most of the unique interactions which are assumed to contribute to the superior protein stability involve electrostatic interactions between groups near the protein surface. The interaction of the Glu 14 side chain with groups on three other residues, Ala 1, Trp 3, and Phe 29 contribute to a more extensive network of main chain to main chain hydrogen bonds. This could only occur in RdPf. Lys 6 is another residue uniquely found in RbPf. It's side chain is observed to form an additional salt bridge with the Glu 49 side chain. There are few differences between the structure of the oxidized and reduced forms of RdPf. These differences are already sufficient to lower the over all stability by $11 \mathrm{deg}$. The exchange of $\mathrm{Fe}$ to $\mathrm{Zn}$ (or Cd) (17) significantly improves the thermal stability although the environment around the hetero-atom is not dramatically changed. Nevertheless the stability rises by $10 \mathrm{deg}$. to $123^{\circ} \mathrm{C}$, whereas the structure and the H-bonding network, as concluded from NMR data, remain unchanged. Unfortunately similar $x$-ray data are at present not available for ferredoxins. But the general conclusions that hyper-thermostability rest on small improvements of the three basic structural features, the main chain to main chain hydrogen bonds, improvements in the hydrophobic core structure, and on crucial exchanges of amino acid residues unique to RdPf will also hold for the ferredoxins. 
CONCLUSIONS: Hyperthermostability is achieved with minimal alterations to the basic protein fold. If we want to improve the stability of newly designed proteins we have to take into account that the hydrophobic core is significantly conserved. Independent of the particular amino acid sequence in the core, and independent of the molecular weight of the protein studied the interactions within the hydrophobic core dwindle and finally vanish when the environmental temperature approaches $113^{\circ} \mathrm{C}$. The improved stability must involve additional electrostatic interactions between groups near the protein surface extending the network of $\mathrm{H}$-bonds.

\section{REFERENCES}

1. Privalov, P.I. (1979) Advan. Protein Chem. 33,167-241

2. Privalov, P.L.\& Gill, St. (1988) Advan. Protein Chem. 39,191-234

3. Murphy,K.P.\& Gill,St. (1990) Thermochim Acta 172,11-20

4. Freire,E.\& Murphy,K.P. (1991) J.Mol Biol.222,687-698

5. Klump, H.,Di Ruggeiro, J., Kessler,M., Park J-B., Adams M.W., \& Robb, F. (1992) J.Biol.Chem.267,22681-22685

6 Adams, M.W. (1990) FEMS Microbiol.Rev.75,125-157

7. Fiala,G. \& Stetter, K.0.(1986) Arch. Microbiol. 145, 56-61

8. Tunicliffe,V. (1992) American Scientist,80,336-349

9. Wachterhauser, G. (1990) Proc.Natl.Acad.Sci.87,200-204

10.Privalov, P.L.\& Khechinashvili,N.N( 1974) J.Mol.Biol.86,665-684

11.Baldwin.R.L. (1986) Proc.Nat1.Acad.Sci. 83,8069-8072

12. Lee, B. (1991) Proc.Natl.Acad.Sci.88,5154-5158

13.Anfinsen, Ch.(1992) personal communication

14.Robb,F.T., Park, J-B. , \& Adams, M.W. (1992) Biochem. Biophys. Acta 1192, 267-72

15.Privalov, P.L. (1982) Advan.Protein Chem. 35,1-104

16. Day, M.W. , Hsu, B. T. , Tor, L-J. Park, J-B, Zhou, Z.H. ,Adams, M.W. \&Rees, D. C. (1992) Protein science, in press.

17. Blake, P. R. , Day, M.W.Hsu, B.T.Tor, L-J. , Park, J-B. , Hare, D. R. , Adams, M. W. , Rees, D.C.\& Summers,M.F.(1992) Protein Science in press. 